\title{
ZIGBEE TECHNOLOGY AND ITS APPLICATION IN WIRELESS HOME AUTOMATION SYSTEMS: A SURVEY
}

\author{
Thoraya Obaid, HaleemahRashed, Ali Abou-Elnour, Muhammad Rehan, \\ Mussab Muhammad Saleh, and Mohammed Tarique \\ Department of Electrical Engineering, Ajman University of Science and Technology \\ P.O. Box 2202, Fujairah, United Arab Emirates (UAE)
}

\begin{abstract}
ABSTARCT
Wireless home automation systems have drawn considerable attentions of the researchers for more than a decade. The major technologies used to implement these systems include Z-Wave, Insteon, Wavenis, Bluetooth, WiFi, and ZigBee. Among these technologies the ZigBee based systems have become very popular because of its low cost and low power consumption. In this paper ZigBee based wireless home automation systems have been addressed. There are two main parts of this paper. In the first part a brief introduction of the ZigBee technology has been presented and in the second part a survey work on the ZigBee based wireless home automation system has been presented. The performances of the ZigBee based systems have also been compared with those of other competing technologies based systems. In addition some future opportunities and challenges of the ZigBee based systems have been listed in this paper.
\end{abstract}

\section{KEYWORDS}

Home automation, ZigBee, Z-Wave, Insteon, Waveins,PAN, voice control, energy management, assistive homes, industrial automation

\section{INTRODUCTION}

Home automation industry has drawn considerable attention of the researchers for more than a decade [1].The main idea is to automatically control and monitor electrical and electronic home appliances. According to the market research firm ABI about 4 million home automation systems were sold globally in 2013 [2]. The same firm also estimated that about 90 million homes would employ home automation systems by the end of 2017. Several commercial and research versions of home automation system have been introduced and built [2-6]. Among these only home security systems have become the main stream of development activities $[7,8]$. Smart home systems have captured several technologies so far and products have been available in the market. Despite over a decade long of disparate activities in the industry companies failed to make home automation as a popular technology. The reasons behind this failure have been comprehensively studied and listed in $[1,9,10]$. Some of these important reasons include cost, difficult to use, vendor dependency, less functionality, and security [11,12]. Moreover, expert hand was required to install, configure, and maintain these systems. Hence, the installation and maintenance costs of the system were high and only rich people with big houses could afford it. In order to overcome some of these limitations wireless home automation system (WHAS) has been introduced and it has gained a considerable attention in the recent years.

The WHAS has reduced the operation and maintenance cost. Additionally, it has provided comfort, security, safety, and remote monitoring capability. A typical WHAS consists of battery operated 
International Journal of Computer Networks \& Communications (IJCNC) Vol.6, No.4, July 2014

low power wireless sensors and actuators attached with the home appliances. These sensors and actuators are connected to a backbone wireless network. The availability of cheap sensors, actuators, and wireless modules has succeeded to make WHAS popular and affordable. It has also reduced the gap between the luxury and mass market segmentations of home automation systems. However, WHAS has some limitations too. These limitations arise due to the hostile radio channel, resource limitation, and mobility. Despite these limitations several organizations and companies have developed WHAS for numerous diversified applications $[13,14]$. Some of the applications include light control, Heating Ventilation and Air Conditioning (HVAC) control, smart surveillance, automated home security, appliance control, smart water supply and irrigation, smart metering, energy regulation, and assistive home. The primary motivations behind these applications include energy saving, improved security, occupant safety, comfortable, and convenient living. The secondary motivations include reducing the operation and maintenance costs of the home appliances, increasing life-time of the same, saving natural resources (i.e., diesel, petroleum etc.), and reducing the greenhouse gases.

The WHAS industry has changed drastically since the introduction of cheap computers and laptops. Moreover, revolutionary developments in the software industry have made the user interface of the WHAS cheap and user friendly [15]. Now-a-days, WHAS can be monitored and controlled from a remote location at any time. There have been many solutions proposed for wireless home automation industry in the past few years. Some of them include Z-wave [16], Insteon [17], Waveins [18], Bluetooth [19], WiFi [20], and ZigBee [21]. In this work we have focused on the ZigBee based WHAS. We have conducted a comprehensive survey work on the ZigBee based WHAS and we presented the summary of this survey work in this paper. This is main contribution of this paper. In addition a comparison of the ZigBee technology based WHAS with other competing technology based systems has also been presented in a later section of this paper. The rest of the paper is organized as follows. Section 2 presents a brief introduction to the ZigBee technology. Section 3 contains a comparison of the ZigBee with other existing technologies. Section 4 contains a comprehensive survey work on the ZigBee based WHAS. Section 5 contains the limitations and challenges of the ZigBee technology. Finally, this paper is concluded with section 6.

\section{The ZigBeE TeChNOLOGY}

A comprehensive description of the ZigBee protocol can be found in the literatures [21,22]. We have presented only a partial description of the ZigBee protocol in this section so that the readers have enough background to understand the rest content of the paper. The ZigBee technology was introduced by the ZigBee Alliance [21]. The ZigBee technology has evolved based on a standardized set of solutions called 'layers'. These optimally designed layers have provided the ZigBee with unique features including low cost, easy implementation, reliable, low power, and high security.

The ZigBee was built on top of IEEE 802.15.4 standard [22]. The IEEE 802.15.4 standard defines the characteristics of the physical and Medium Access Control (MAC) layers for Wireless Personal Area Network (WPAN). Taking this standard as a "chassis" the ZigBee Alliance has defined the upper layers in the ZigBee standard. Devices are the main components of the WPAN. The devices have been categorically defined as (a) physical type, and (b) logical type. The physical type devices have been further classified into two types namely Full Function Device (FFD) and Reduced Function Device (RFD). Any device may act as a sensor node, control node, and composite device irrespective of its type. Only the routing functions of a network are performed by the FFDs. Depending on their locations in a network the FFDs may have one or more child devices and they perform routing functions for these child devices. The RFDs do not perform routing function in a network and hence they cannot have any child device. 
The logical type devices have been further classified as three types namely coordinator, router, and end device. Among these logical devices the coordinator is the most capable device, which forms the root of the network tree. There should be exactly one ZigBee coordinator in a network to initiate the formation of a network tree. It also acts as a bridge to other networks. The ZigBee end devices possess limited functionality to communicate with a coordinator or a router only; it cannot relay data for other devices. Due to this limited functionality the end devices can "sleep" for a significant amount of the time and hence can enjoy a long operating life.

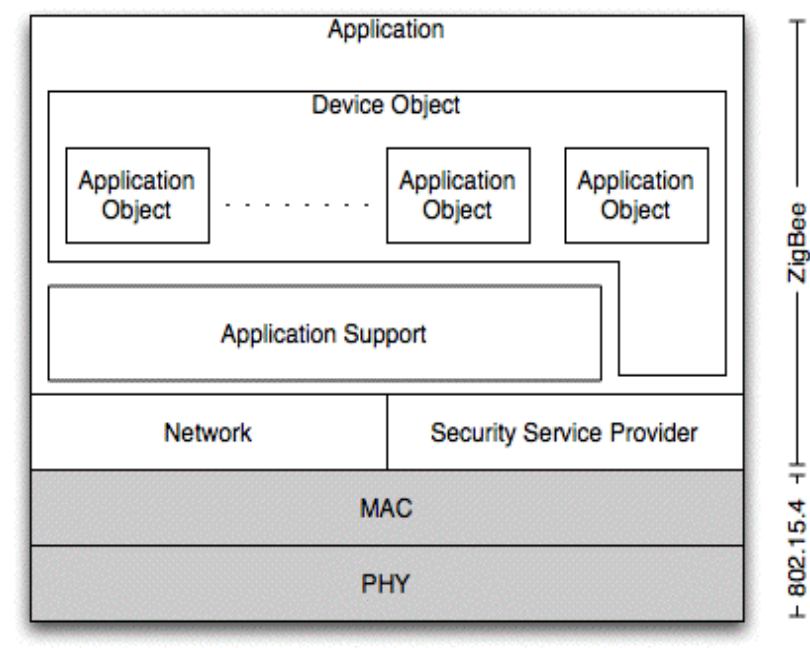

Figure 1: The ZigBee and IEEE 802.15.4

The protocol stacks defined by the ZigBee Alliance with respect to IEEE 802.15.5 standard protocol stacks are shown in Figure 1. The ZigBee architecture includes the Application Support (APS) sub-layer, ZigBee Device Object (ZDO), and user-defined application profile(s). The APS sub-layer's responsibilities include maintenance of some tables, which contain information used to enable matching and establish communication among the devices. During the discovery phase these tables are also used by a device to identify other devices that operate in the operating space. The ZDO determines the nature of the device (i.e., coordinator or FFD or RFD) in a network. It also replies to binding requests while ensuring asecured relationship between two devices. The user defined application refers to the end device that conforms to the ZigBee Standard. 


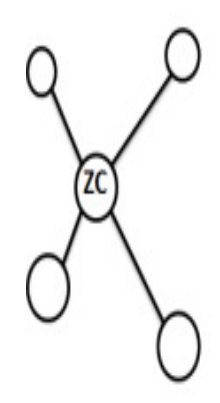

(a)

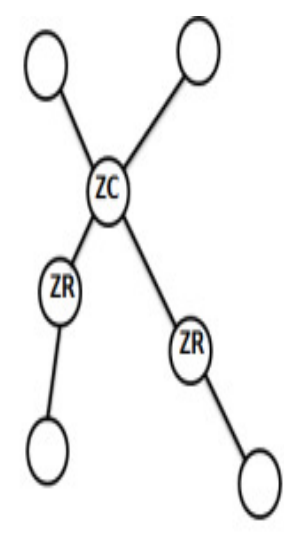

(b)

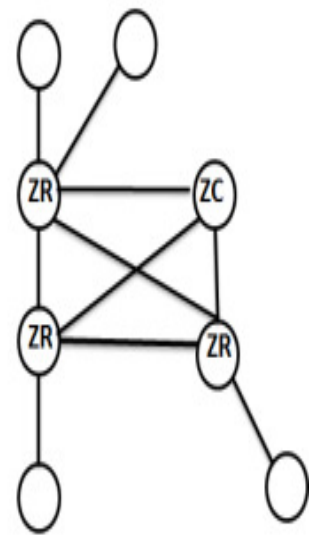

(c)

Figure 2 Network topologies of ZigBee network (a) star, (b) Tree, (c) mesh

The network layer assists the network to grow. This layer can handle a network consisting of up to 64000 nodes. The physical layer accommodates a high level of integration by using direct sequence technique. The Medium Access Control (MAC) layer permits to form several topologies without introducing complexity. The ZigBee devices have 64-bit addresses, with an option to enable shorter addresses to reduce packet size, and work in either of the two addressing modes namely star and peer-to-peer as shown in Figure 2. Some of the other technological features of the ZigBee have been listed in Table 1.

Table 1: Features of the ZigBee technology

\begin{tabular}{|l|l|}
\hline Parameter & Value \\
\hline Data Rate & $250 \mathrm{~kb} / \mathrm{s}, 40 \mathrm{~kb} / \mathrm{s}$, and $20 \mathrm{~kb} / \mathrm{s}$ \\
\hline Topology & Star or Peer-to-Peer \\
\hline Addressing & 16 -bit (short) or 64-bit (extend) \\
\hline Multiple Access Technique & Carrier Sense Multiple Access with \\
& Collision Avoidance (CSMA/CA) \\
\hline Frequency & 868 (Europe) \\
& $915 \mathrm{MHz}$ (North America) \\
& $2.4 \mathrm{GHz}$ (Worldwide) \\
\hline Range & $10-20$ meter \\
\hline Channels & 11 channels $(868 / 915 \mathrm{MHz} 0$ \\
& 16 channels $(2.4 \mathrm{GHz})$ \\
\hline
\end{tabular}

Two modes of communication are used in a ZigBee network namely (a) beacon mode, and (b) nonbeacon mode. The beacon mode is used by a battery operated coordinator to save power. A device waits for the beacons that are periodically transmitted by the coordinator and looks for the messages addressed to it. If the message transmission is complete, the coordinator sets a schedule for the next beacon for this device. After knowing the schedule for the next schedule the device can go to sleep. On the other hand, the non-beacon mode is used by a mains-powered coordinator. All devices in a mesh network know the schedule to communicate with each other and they need to wake up at a scheduled time so that they do not miss the beacon. Hence, a quite accurate timing circuit needs to be associated with the devices. It means that there will be an increase in power consumption. The non-beacon mode communication is suitable for applications such as smoke 
International Journal of Computer Networks \& Communications (IJCNC) Vol.6, No.4, July 2014

detector and burglar alarm applications where devices 'sleep' nearly almost all the time. Till now ZigBee has found numerous applications. Some of these applications include smart energy network, home entertainment and control, monitoring and controlling industrial plant, health care, and home automation. In this work, we limit this effort only to the home automation system application.

\section{RELATED TECHNOLOGIES}

The competing technologies of the ZigBee are Z-wave, Insteon, Waveins, Bluetooth, and WiFi. A comparison of these technologies has been presented in Table 2.

Table 2: Comparison of different WHAS technologies

\begin{tabular}{|c|c|c|c|c|c|c|}
\hline Technology & Z-Wave & Insteon & Waveins & Bluetooth & WiFi & ZigBee \\
\hline Frequency & $\begin{array}{l}868 \mathrm{MHz} \\
908 \mathrm{MH} \\
2.4 \mathrm{GHz}\end{array}$ & $904 \mathrm{MHz}$ & $\begin{array}{l}433 \mathrm{MHz} \\
868 \mathrm{MHz} \\
915 \mathrm{MHz}\end{array}$ & $2.4 \mathrm{GHz}$ & $\begin{array}{l}2.4 \mathrm{GHz} \\
5 \mathrm{GHz}\end{array}$ & $\begin{array}{l}868 \mathrm{MHz} \\
915 \mathrm{MHz} \\
2.4 \mathrm{GHz}\end{array}$ \\
\hline Modulation & FSK/GFSK & FSK & GFSK/PSK & FHSS & $\begin{array}{l}\text { QPSK } \\
\text { COFDM } \\
\text { QAM }\end{array}$ & $\begin{array}{l}\text { BPSK } \\
\text { O-QPSK }\end{array}$ \\
\hline Error Control & CRC(8-bit) & CHECKSUM & $\mathrm{BCH}$ & CRC (16- bit) & CRC(32-bit) & CRC(16-bit) \\
\hline Range & $30-100 \mathrm{~m}$ & $45 \mathrm{~m}$ & $200-1000 \mathrm{~m}$ & $10 \mathrm{~m}$ & $100 \mathrm{~m}$ & $10 \mathrm{~m}-100 \mathrm{~m}$ \\
\hline Network size & 232 & 256 & unknown & 8 & 2007 & 64000 \\
\hline $\begin{array}{l}\text { Power } \\
\text { Consumption }\end{array}$ & Low power & NA & Ultra-low & Medium & High & Very Low \\
\hline
\end{tabular}

The Z-wave is a wireless solution developed by Sigma Design and it is promoted by the Z-wave alliance [16]. The main applications of the Z-wave are in residential and light commercial environment. The purpose of the Z-Wave is to support a reliable transmission of short messages from a control unit to one or more nodes in a network. The Z-Wave network operates in the 900 MHz ISM band. It can support a data rate up to $64 \mathrm{kbps}$ by using Binary Frequency Shift Keying (BFSK) modulation.

Insteon is a home automation solution developed by Smart Labs and promoted by the Insteon Alliance [17]. The Insteon defines mesh topology by using radio frequency (RF) link and power line link. The nodes can support either RF links or power line links. They can also support both types of communication. Insteon operates in the $904 \mathrm{MHz}$ frequency band. It can support a data rate up to 38.4 kbps by using Frequency Shift Keying (FSK) modulation. It also supports multihop 
communication in order to increase the coverage area. But, the maximum number of hops that data can travel is limited to four.

Waveins is a low power wireless protocol developed for controlling and monitoring home appliances. It is currently managed and promoted by Wavenis Open Standard Alliance [18]. This protocol defines physical, link, and network layers. Wavenis services can be accessed from the upper layer through an application programming interface. Like Insteon the operating frequencies of Waveins are in the ISM Band (i.e., $433 \mathrm{MHz}, 868 \mathrm{MHz}$, and $915 \mathrm{MHz}$ in Asia, Europe, and United States respectively). The maximum data rate offered by Waveins is 100 kbps. Gaussian Frequency Shift Keying (GFSK) and Fast Frequency Hopping Spread Spectrum (FHSS) radio technologies are used in Waveins.

Bluetooth, a standard maintained by Bluetooth Special Interest Group (SIG), primarily designed for short range Personal Area Network (PAN) applications [19]. It uses low power and low cost transceiver. Bluetooth uses a special radio technology called Frequency Hopping Spread Spectrum (FHSS), which uses up to 79 frequencies during the hopping. Bluetooth provides a way to connect and exchange information among devices including mobile phones, telephones, laptops, personal computers, digital cameras, and video game consoles.

WiFi was introduced by WiFi Alliance [20]. The main target of WiFi was to replace the wires of local area network (LAN) and hence to reduce the network operation and maintenance costs. Almost all certified products that belong to a wireless local area network may be connected to a WiFi network. The ever dropping cost of wireless chipsets for WiFi network has helped this technology to occupy a mass market segmentation of the wireless industry. The WiFi technology is backward compatible and it is a global set of standard. Hence, a compatible device can be connected to a WiFi network located anywhere in the world. The operating range of WiFi network is very limited (i.e., 32 meter indoors and 95 meter outdoors). The range of WiFi network is the main cause of its limited applications that include inventory tracking machine, bar code reading, and retailing.

There are also some other emerging home automation technologies that compete with the ZigBee technology. One of them is the IP-Based solution, which has been initiated by the Low Power Wireless Personal Area Network Group of Internet Engineering Task Force (IETF). This working group has defined mechanism for transmission of IPv6 packets on top of IEEE 802.15.4 networks. This type of network has been named as LowPAN [23]. The LowPAN follows the mesh topology and a routing protocol is used for its operation. The work on LowPAN is still in its infancy level and it is predicted that it will be an emerging technology for WHAS in a near future.

Another home automation technology, originated in Germany, is EnOcean. This technology has been introduced by Enocean Alliance [24] for building automation and it has been standardized as ISO/IEC 14543-3-10 in 2012. This standard is based on the philosophy of "No Wire, No Battery, and No Limit". This standard defines the physical, data link, and network layer. The EnOcean uses an innovative technology called 'energy harvesting' because in this technology a device generates its own energy to operate. Hence, the EnOcean compliant sensors and light switches can operate without batteries. This main advantage of the EnOcean has opened up a huge potential market in the automation industry. The radio signals from these sensors and switches can be transmitted wirelessly over a distance of up to 30-300 meters depending on the locations of deployment. The transmission frequencies used for the devices are $902 \mathrm{MHz}, 868.3 \mathrm{MHz}$, and $315 \mathrm{MHz}$.

Ultra-wideband (UWB) [25] is also a potential candidate for WHAS. Like the ZigBee this technology also consume a very low power. Originally, the UWB was introduced for radar imaging. But, it is now considered a suitable technology for other applications like target sensor data collection, precision locating, and tracking applications. It can transmit information which is 
International Journal of Computer Networks \& Communications (IJCNC) Vol.6, No.4, July 2014

spread over a large bandwidth ( $>500 \mathrm{MHz})$. The UWB can support a network consisting of up to 8 devices.

\section{The ZigBee Based Wireless Home Automation Systems}

In this section the survey work on the ZigBee based WHAS is presented. Many applications of the ZigBee based WHAS have been proposed in the literatures. These applications can be loosely categorized into four major classes namely (a) security[26-28], (b) smart surveillance [29-34], (c) energy management [35-39], and (d) assistive home [40-47].

In security applications WHAS has been proposed to ensure security of a home. One of the early experimental ZigBee based home security system has been proposed in [26]. This system is capable of monitoring door and window, smoke, gas leak, and water flooding in a home from a remote location. Some simple control systems (i.e., operating a valve and sending signal to security network) have also been associated with this application. The security alarming system has been implemented by using ZigBee chip called MC13192 and low power consumption micro-controller called MSP430F135. The system also supports Web interface so that a user can access the system remotely to control, search, and review record. The proposed system was configured by using an LCD panel.

Another similar system has been proposed in [27].The proposed system has been developed for automatic door opening and closing, temperature monitoring, gas detection, and light control. Three technologies namely RFID, ZigBee, and GSM have been used to implement the system. Controlling light and fan has been implemented by using GSM so that these home appliances consume minimum power. Temperature monitoring system has been developed by using GSM technology.

Intelligent home automation system (IHAM) has been presented in [28] to ensure security at home. The system uses PIC microcontroller with ZigBee wireless communication technology, speech recognition technique, and GSM network. The home automation system is used to control all lights and electrical appliances in a home or office using voice commands with the help of HM2007 chip. In this work the authors have presented the overall framework for hardware and software to implement the system. The proposed system has also integrated a security warning system with it so that the users can be warned about the fire hazards. This warning system has been implemented by using a smoke sensor and GSM Module. The system is able to send an SMS to the user if smoke is detected.

In intelligent surveillance applications WHAS has been used to monitor the activities at home. Some of these systems also allow a user to do the same from a remote location. One such system based on the ZigBee has been designed in [29]. The system transmits periodic data from a location information service to determine the current position of a user. The proposed system consists of three components namely indoor position system (IPS-M), indoor position system-infrastructure (IPS-I), and indoor position system-gateway (IPS-G). The IPS-I device periodically transmits active beacons to an IPS-M device to estimate distances. The authors have claimed that the proposed system performs better than a passive mobile system. The IPS-M receives wireless signals from an IPS-I to track an IPS-M.

In a similar work [30] Digital Living Network Alliance (DLNA) compliant digital home appliances have been addressed. According to the authors DLNA compliant devices will be become widespread in the near future. Hence controlling these devices by using sensors via some networks has become an important issue now. The authors have also claimed that the ZigBee technology can be considered a suitable solution for such type of network. To interconnect DLNA compliant home 
International Journal of Computer Networks \& Communications (IJCNC) Vol.6, No.4, July 2014

appliances with a ZigBee network a Gateway is necessary. The authors have proposed the architecture of such Gateway. They also proposed an energy efficient method for controlling the sensor network. This kind of energy efficiency has been achieved by using dynamic switching between unicast and broadcast in the sensor data collection method. The authors have proved that the proposed system is power efficient by measuring the power consumption in the network.

Another home network gateway based on the ZigBee technology has been proposed in [31]. The system has realized the connection of low rate wireless home network and Internet. A user can control the home appliances through the proposed home network gateway. This was one of the earliest works to depict that the home appliances could be controlled from a remote location via Internet. The hardware and software used to design the home gateway and device nodes have also been presented in the same work.

Another similar wireless remote monitoring for home security has been proposed in [32]. In this work a real time surveillance of the home security was developed based on variety of sensors, the ZigBee technology, and GSM/GPRS network. The proposed system can send abnormal images and warning messages through MMS and SMS. The system can also receive remote instruction to monitor and control the household appliances. The reliability of the proposed system has been tested and the authors have claimed that the proposed system can successfully guarantee home security for a remote user. The experimental result shows that the system has remote surveillance capability to ensure home safety with high availability and reliability.

A ZigBee wireless sensor networks of star topology has been proposed for wireless intelligent home system in [33]. The proposed system is suitable for a typical small home network. The system can be remotely monitored and controlled by using GSM module. The system is composed of the following three main components: (i) home server with GSM module, (ii) intelligent environment detection sensor modules, and (iii) intelligent home appliances. The concepts and the architectures of the proposed system have been discussed in the same work. The system has been tested to ensure its remote alarming and control ability.

Another wireless home automation system based on the Internet of Things and the ZigBee wireless sensor network technology has been proposed in [34]. The authors have implemented the system by using Texas Instruments MCU device LM3S9B96, which is based on the ARM Cortex-M3 based controller. The entire system can run on the $\mu \mathrm{C} / \mathrm{OS}$-II embedded real-time multitasking operating system. Users can access this system by using a dynamic webpage of LwIP TCP/IP protocol stack or GSM SMS. By using this system a user can monitor and control the environmental parameters such as temperature, humidity, meter readings, and light of a home. The system is also able to monitor and control the home appliances like light, air conditioner, and heater.

In energy management applications WHAS has been used to save energy consumed in a home. This energy saving is achieved by controlling the electrical and electronic home appliances. One such ZigBee based power monitoring system (PMS) has been reported in [35]. In addition to ZigBee wireless communication the PMS also utilizes Digital Signal Processing (DSP), and Web services. This work possesses the novelty of integrating ZigBee, DSP, and Web Services technologies together. The DSP has been used for the computation of real-time power parameters. The Web Services are used for the communication infrastructure among distributed systems across a network. The proposed system has been constructed, tested, and validated for the power management of a campus. The test results show that the functions of the proposed PMS comply with the designed objectives. The proposed system also demonstrates good performances in direct load control and in transmission of warning message. 
Some of the ZigBee based energy efficient system has been targeted to control the power of electric outlets for saving energy. One such work has been proposed in [36]. The proposed system can also measure the currents drawn by electric outlets. The system has been implemented by using an embedded board and the ZigBee technology. This proposed system has two main components namely ZigBee control module and the server module. The ZigBee control module consists of several controllable outlets, a current measurement circuit, a ZigBee transmitter, a ZigBee receiver, and a micro control unit. The measurement circuit measures the current drawn by the electric outlets and it sends a signal to the server module through the ZigBee transmitter. The data of the current and voltage are stored in an embedded board. The proposed system can detect any overload and can send a message to the circuit breaker to safely turn OFF the power. Visual Basic has been used to design the user interface so that a user can easily operate the system.

In order to overcome the architectural limitations of wireless sensor network a ZigBee based intelligent self-adjusting sensor (ZiSAS) has been introduced in [37]. Due to the architectural constraints of wireless sensor networks there is a trade-off between the performance and cost. Sometimes, wireless sensor based home automation system cannot be effectively implemented in home environments. In order to overcome this limitation ZiSAS has been proposed. The ZiSAS uses a situation-based self-adjusting scheme. Hence, ZiSAS is an event driven self-adjusting sensor network implemented by using hardware and middleware. The proposed system has been tested in a real test-bed. The experimental results show that the proposed system reduces energy consumption in a home.

In many industrial applications the cost of a wireless sensor network is not an important factor. It is more important to deploy the network in rapidly changing application environments and design requirements. In this regard two major issues are (a) to rapidly construct application software for different design requirements, and (b) to operate the system smoothly. One such rapidly deployable system has been reported in [38]. An automatic embedded software generation framework has been proposed in this work that can rapidly create and evolve ZigBee applications. The framework consists of several major modules namely (a) pattern extraction, (b) code generation, and (c) architecture mapping. The authors have provided an embedded software development framework that integrates the heterogeneous readers and sensors interfaces with an optimal energy control model to enhance the quality of digital home living environments. The proposed framework allows rapid deployment of the supporting software for a suite of energy control and sensing devices aiming not only at energy saving but allowing individual residents to monitor their energy usage at any time. The authors have claimed that the proposed tool has successfully completed the goal to control the comfort level at home with minimum amount of energy consumption.

In [39] the authors have suggested the requirements for an appropriate technology for automation system. They have claimed that selection of an appropriate technology for home and industrial automation systems should be based on low cost, easy placement and installation, easy extension, comfort benefits, and mobile device connectivity. An energy aware home automation system satisfying all these requirements have been proposed in [39].The proposed system can control load and hence can save energy. This ZigBee based system is used for remote controlling and monitoring of various home loads/appliances. The ultimate objective is efficient power utilization through real time power level indicator with the help of a PC-based GUI application. The authors have investigated various performance parameters such as latency, received signal strength indicator (RSSI) value, and round-trip delay time (RTD). They also have compared the proposed ZigBee based system with that of a Bluetooth based system. The results show that the ZigBee based system can save more energy compared to its Bluetooth counterpart. A comparative study of different wireless 
protocols such as ZigBee (over IEEE 802.15.4) and Bluetooth (over IEEE 802.15.1) has been investigated in the same work. The objective of this work is to investigate the suitability of different technologies and select the best one for home automation. The home automation system presented in this work is based on ZigBee has been proposed for remote controlling and monitoring of various loads/appliances. The ultimate objective is to ensure efficient power utilization through real-time power level indicator with the help of a PC-based GUI application. The authors have claimed that the ZigBee based system performs better compared to its Bluetooth counterpart in terms of latency, RSSI value, and RTD.

In assistive applications WHAS has been proposed to assist the old people and people with disability. One such ZigBee based assistive WHAS has been presented in [40]. The proposed system is based on a voice control system. The system has integrated a voice recognition module with a ZigBee based network. The recognized messages sent by the module are routed to electronic devices located in the home. In this work SI-ASR (Speaker-Independent Automatic Speech Recognition) has been used. The proposed system does not require any training and recording of the words. This speech recognition system has been used to implement the human computer interaction to realize multiple menu selection functions. A novel speech recognition method has been used for ordering songs stored in a SD memory card. To improve the accuracy of the system three control methods have been proposed namely speech recognition control, button trigger mode, voice password trigger mode, and circle recognition mode. A user can choose a mode depending on the condition. A prototype of speech recognition module has also been presented in the same work. The authors have provided some experimental results to validate the basic functions of the proposed system. It has been shown that the system is easy and flexible for people, especially the old people and people with disabilities.

A low power voice control system for home automation system has been proposed in [41]. The motivation behind this work is to provide supporting systems for the elderly and the disabled people. The system is based on the recognition of voice commands and it uses low-power RF ZigBee wireless communication module. The home automation system is intended to control all lights and electrical appliances in a home and office using voice commands. The ZigBee technology has been used to receive the voice command and send the voice data to an ARM9 controller. The controller then converts the voice into a required format and sends the data through a ZigBee network to another ZigBee module and micro controller attached with the devices. Based on the received message the system can either turn ON or OFF the devices. The proposed system has been extended to include a multimedia streaming, in which Differential Pulse Code Modulation (DPCM) compression algorithm has been used to compress the speech data by half.

Another foolproof solution to control the home appliances via voice commands for physically challenged people has been proposed in [42]. In the proposed system the voice command is sent wirelessly to a personal computer. A fault identification system has been incorporated in the proposed system to monitor the exact status of the home appliances. By using this fault detection system a user can ensure that the home appliances had gone exactly ON or OFF or undergone fault. The authors claimed that the proposed system has some advantages because it receives status information from the load end. But, the other previously proposed systems get the status from the user end. This end user data may provide a false indication when power supply is not available for a particular load or when load get open circuited due to wire discontinuity or open fuse condition. User screen navigation, voice output of the current screen information, and status of the automated appliances enable visually impaired person to control their home appliances. Navigation of the screen by using voice commands enables person with disability to control their home loads like a normal person. Low cost ZigBee has been usedto provide wireless environment and RFID has been used to provide secured authentication. Each home load is controlled by two commands namely ON and OFF. The proposed system has been tested to handle 20 loads such as mixer, grinder, TV, refrigerator, fan, light, and air conditioner. Forty voice commands have been used to test the 
International Journal of Computer Networks \& Communications (IJCNC) Vol.6, No.4, July 2014

system. When a user creates own profile and automates the load, the speech recognition system can successfully recognize the voice with an accuracy of more than ninety percent. The user can allow other people to use the system with the same profile. The success rate for this case is seventy five percent.

Another similar ZigBee based voice controlled WHAS has been proposed in [43]. The system is based on recognition of voice commands and it uses low-power RF ZigBee wireless communication modules. The home automation system is intended to control all lights and electrical appliances in homes and offices using voice commands. The verification tests include voice recognition response test, indoor ZigBee communication test, and the compression and decompression tests of DPCM speech signals. The tests involved a mix of 35 male and female subjected to different English accents. Thirty five different voice commands were sent by each person. Thus the test involved sending a total of 1225 commands and $79.8 \%$ of these commands were recognized correctly.

In another recent work [44] a voice controlled ZigBee based secured home automation system has been proposed for people who are living alone and who are living with disability. In the proposed system a voice recognition chip HM 2007 has been used. The chip can recognize 20 words and each word has duration of 1.92 second. The 8051 microcontroller (AT89S51) has been used to implement the system. The authors also claimed that the proposed system is itself a secured one because of the voice recognition system.

Another similar work has been presented in [45].The proposed system in [45] allows controlling of devices using voice commands and reduces user interaction with system directly. This System uses SAPI (Speech Application Programming Interface), which is a Microsoft Application to enable voice recognition. This system contains three main components: (i) intelligent home server with ZigBee module, (ii) intelligent environment detection sensor modules, and (iii) voice command controlling module. The various features of the system include turning any home appliances or devices, playing media applications, downloading RSS feeds, and sending mail. The authors also proposed some architecture to connect the system with the internet so that any device eventually can be used to control and monitor the devices.

A voice command and touch screen based home automation system has been proposed in [46]. The system has been tested and verified. The verification tests include voice recognition response test, touch screen response test, and indoor ZigBee communication test. A touch of symbol on screen can control the home appliances using the ZigBee technology. The home devices can be operated remotely. It can be used at home, industry, hotels, shopping malls, and process control systems. This system is mainly implemented for multi-disabled people using voice recognition and touch screen technology. Automatic Speech Recognition (ASR) engines through Microsoft speech APIs has been used in this system. The system is implemented by using TFT touch screen.

A similar system has been built and implemented in [47]. The proposed system can recognize voice commands and it uses low-power RF ZigBee wireless communication modules to control the lights and electrical appliances in a home and office using voice commands. The system has been tested and verified. The tests involved a mix of ten male and female subjects with different languages. Seven different voice commands were sent by each person. The test results involved sending a total of 70 commands and $80.05 \%$ of these commands were recognized correctly. 
International Journal of Computer Networks \& Communications (IJCNC) Vol.6, No.4, July 2014

\section{The Challenges of ZigBee Based Home Automation Systems}

Although the ZigBee technology is considered as the most popular technology for WHAS, it has some challenges too. Some of the challenges are as follows:

- Resource Constraint: The sensors (or nodes) used in WHAS have limited resources (i.e., limited processing power, low memory, and limited battery).

- Limited Range: The ZigBee has limited transmission range and multihop communication is required to increase the range of a ZigBee network.

- Technological Limitation: The ZigBee technology depends on the physical layer and medium access control layer of IEEE 802.15.4 standard.

- Interferences: The WHAS has to co-exist with other systems operating in the same free band called Industrial Scientific and Medical (ISM) bands. Hence the communication of WHAS can be interfered by other systems (i.e., Bluetooth, WiFi, cordless phone, and microwave oven) operating in the same frequency band.

- Internet Connectivity: For remote monitoring the WHAS may need to have internet connectivity.

In order to cope with the resource constraint problem nodes (or sensor) with higher processing power need to be used in WHAS. By using existing ZigBee technology the resource constraint problem can also be solved to some extent. As mentioned before ZigBee defines two device types namely RFD and FFD. The FFDs have higher processing power compared to the RFDs. Hence, to overcome the resource constraints a WHAS should include more FFD devices and few RFD devices. The limited battery constraint is not a major issue for ZigBee technology because the ZigBee device consumes less power compared to other competing technologies like Bluetooth and WiFi. Hence, the ZigBee based WHAS lasts longer compared to other technologies based WHAS. It has been claimed in [48] that the operating life of a typical ZigBee network is 100-1000 days. On the hand the operating life of $\mathrm{WiFi}$ is 5-10 days and the same for Bluetooth is 1-7 days. There have been a number of published investigations on this issue. One of these investigations has been reported in [49]. In this work the performances of a ZigBee based WHAS have been compared with those of WiFi and Bluetooth based WHAS. The authors argued that many home automation systems use WiFi and Bluetooth for WHAS. But, the power consumption is very high for these two technologies. This kind of high power consumption has made these two technologies unsuitable for WHAS applications, where a longer operating life is highly desirable. The experimental results published in [49] show that the ZigBee technology based WHAS can operate for longer time compared to its counterparts. The authors have suggested the ZigBee based WHAS for the applications, where longer operating life is an important issue.

The limited range of the ZigBee based WHAS can be easily extended by using ZigBee router. The ZigBee Alliance has already defined the functionalities for a ZigBee router. According to the ZigBee specifications a FFD device can be configured as a router. Moreover, the ZigBee based WHAS can be easily connected to the internet, which in turn overcomes its limited range constraint. Some recent investigations have been conducted to extend the range of a ZigBee network. In [50] the investigators have proposed the SKY65336 and SKY65337 ZigBee front-end modules to extend the range of a ZigBee device. It has been shown that the range of a ZigBee device can be extended up to 543 meter and 923 meter by using the SKY65336 and SKY65337 modules respectively. Some amplifiers are already in market that can also be used with a ZigBee device to increase its range up to 4000 meter [51]. 
International Journal of Computer Networks \& Communications (IJCNC) Vol.6, No.4, July 2014

One major technological limitation of the ZigBee protocol arises from its dependency on the IEEE 802.15.4 standard upon which the ZigBee networking and applications are hosted in. The ZigBee Alliance does not have any control on the IEEE 802.15.4 standard. However, the people who served in the IEEE 802.15 Working Group are also deeply involved in designing the ZigBee standard. A close tie is required between the ZigBee Alliance and IEEE 802.15.4 Standardization Committee to keep track of the changes made in the IEEE 802.15.4 standard in future.

The coexistence of the ZigBee based WHAS with other system is also an issue. This issue has been addressed by the researchers in several works. One such type of investigation has been reported in [52]. In that work the authors have argued that WHAS operates in a microwave radio band, which may be shared by several home appliances in a home. For example, laptops, wireless routers, cordless phones, and microwave ovens occupy the 2.4 $\mathrm{GHz}$ band that is located in the same operating band of WHAS. Hence, the interoperability of these home appliances with the WHAS presents a significant challenge for the home automation system designer. The authors have performed several laboratory experiments to study the coexistence of WHAS with wireless card equipped laptop and microwave oven. The system performance has been evaluated based on the measurement of Packet Delivery Ratio (PDR) metric. The results show that the presence of the home appliances significantly affects the performance of WHAS. A solution to this problem has been reported in [53]. In that investigation a ZigBee based home automation system and a WiFi based network have been integrated through a common home gateway. The home gateway provides network interoperability, a simple and flexible user interface, and remote access to the system. The same work has shown that a successful co-existence and interoperability of Wi-Fi and ZigBee in a real home automation system is possible if the system is carefully designed.

In order to connect the ZigBee based WHAS with the internet a suitable gateway is a must. Several researches have been carried to design such gateways. Two-tier architecture of home control system (HCS) based on ZigBee multi-hop mesh network has been presented in [54]. In this work the basic data acquisition, processing functions, and the architecture of the proposed gateway have been designed and fabricated. The proposed gateway supports three interfaces namely (a) ZigBee based wireless access interface, (b) Bluetooth based local interface, and (c) GPRS based remote interface. The hardware architecture and protocol software as well as the communication control method have been proposed for this gateway. The proposed gateway's performances have been tested under a constructed test environment. The performance results show that the proposed gateway is convenient for WHAS.

A similar work has been presented in [55], which is a ZigBee based embedded remote control system. The system also runs on an embedded board rather than on a PC. The paper describes the running and configuration procedures of the system on the embedded board. Since the embedded Web Server uses common gateway interface (CGI) to communicate with other modules in the system, the CGI program is also used in this work. The software design of the ZigBee is based on TI's Z-stack, and the ZigBee modules are implemented by TI's CC2430. The testing system can be used from any remote places via an Internet interface and a browser. The control modules can be communicated via wireless mode. Therefore, the proposed system can be considered as a promising candidate for home automation applications, remote industrial control areas, and remote patient monitoring system.

Another gateway has been proposed in [56] where an automation system has been designed to switch ON, OFF and get to know the status of home appliances by using ZigBee from a remote location. The home appliances are connected to the ports of a microcontroller board and their status are passed to the home server. The combination of NetBeans and Embedded $\mathrm{C}$ is used for 
monitoring and controlling software. The home appliances can be monitored and accessed remotely by a user via SMS or EMAIL.

In addition to the above mentioned constraints the ZigBee based WHAS has some other problems too. Orphan node is one of them [57]. This problem arises because of the addressing scheme used in the ZigBee technology. In ZigBee, a device (with a permanent 64-bit MAC address) is said to join a network when it can successfully obtain a 16-bit network address from a parent device. Parent devices calculate addresses for their child devices by a distributed addressing assignment scheme. This assignment is easy to implement, but it restricts the number of children of a device and the depth of a network. The ZigBee address assignment policy is too conservative and thus usually making the utilization of the address pool poor. Those devices that cannot receive network addresses will be isolated from the network and become orphan nodes. A solution to this problem has been proposed in [58].

Like any other wireless system the ZigBee network has security issues and this issue is very important for the ZigBee based WHAS application where security is an important issue (i.e., home security system). The ZigBee is unreliable to various wireless mesh protocol network attacks and penetration. Other drawback of the ZigBee based WHAS is the cost of installation. The current homes must be retrofitted somewhat to accommodate ZigBee, which may drive initial costs up.

Although the ZigBee based WHAS has still some limitations to be solved, it is still considered more suitable technology for WHAS compared to other technologies. Several investigations have already proved this claim. For example, one major advantage of the ZigBee is that it can be easily interfaced with different types of microcontroller. This issue has been addressed in [59]. In this work different microcontrollers have been used in a ZigBee based WHAS. Four popular microcontrollers were used to investigate memory requirements and power consumption such as ARM, x51, HCS08, and Coldfire. The authors have claimed that the ZigBee technology is interoperable with these microcontrollers and this is an essential feature of WHAS. The interoperability of the ZigBee and other microcontrollers has also been investigated in [60]. A system consists of different relays, ZigBee module CC2520, and microcontroller AT89S52. The authors also investigated the data rate and coverage area in indoor and outdoor applications. They also have compared the performances of the proposed system with that of another system implemented by Bluetooth. The authors have shown that the ZigBee based system outperforms the other Bluetooth based system.

\section{Conclusions}

In this work, a technical overview of the ZigBee technology has been presented. The main features of the ZigBee technology have been highlighted in this paper. Although the ZigBee has numerous applications, we limit this effort only to its application in WHAS. The performances of the ZigBee based WHAS have been compared with those of other competing technologies including Z-Wave, Insteon, Waveins, WiFi, and Bluetooth. It has been shown that the ZigBee based WHAS outperforms other technology based WHAS. A comprehensive survey work on the ZigBee based WHAS has been presented in this paper. Some limitations and challenges of the ZigBee based WHAS have also been listed in this paper. Based on this survey work we can conclude that the ZigBee can be considered as the most suitable technology WHAS compared to other existing technologies. But, there are still some challenges of ZigBee based WHAS that are still under investigations. 
International Journal of Computer Networks \& Communications (IJCNC) Vol.6, No.4, July 2014

\section{References}

[1] A.J. Bernheim Brush, Bongshin Lee, Ratul Mahajan, Sharad Agrawal, Stefan Saroiu, Collin Dixon,(2011) ,"Home Automation in the Wild: Challenges and Opportunities", Proceedings of ACM CHI Conference on Human Factors o Computing System, May 7-12, Vancouver, BC, Canada

[2] ABI Research on home automation available at http://www.abirearch.com

[3] Richard Harper, "Inside the Smart Home", Springer-Verlag London Limited (2003)

[4] Juile A Kietz, Shwetak N. Patel, Brian Jones, Ed Price, Elizabeth D. Mynatt, Greogory A. Abowd, (2008) "The Georgia Tech aware Home", The 26th CHI Conference, April 5-10, Florence, Italy, pp. 3675-3680

[5] TiiuKoskela and Kaosa-vaananen-vainio-Mattila, (2004) "Evolution towards smart home environments:empirical evaluation of three users interfaces, International Journal of Personal and Ubiquitous Computing, July, Vol. 8, No. 3-4, pp. 234-240

[6] Daine J. Cook and Sajal K. Das, (2005) "Smart Home Environments: Technology, Protocols, andapplications", Wiley series of Parallel and Distributed Computing (kindle Edition, pp. 273-294

[7] Tom Rodden and Steve Benford,(2003) " The evolution of buildings and implications for the design of ubiquitous domestic environments", Proceedings of the SIGCHI Conference on Human Factors in Computing Systems, April 5-10, Fort Lauderdale, Florida, pp. 9-16

[8] Home security systems, home security products, and home alarm systems available at http://www.adt.com

[9] W. Keith Edwards, Rebecaa E. Grinter, Ratul Mahajan, and David Wetherall,(2011)“ Advancing thestate of home networking", Communication of ACM, Vol. 5, No.6, July, pp. 62-71

[10] Forest and Sullivan, "North American Home Automation Market", available at http://www.forest.com/sublib

[11] Intile S.S., (2002) "Designing a home of the future", IEEE Pervasive Computing, Vol. 1, No.2, pp. 7682

[12] Scott Davidoff, Min Kyung Lee, Charles Yiu, John Zimmerman, and Anind K. Dey,(2006) “ Principlesof smart home control", Proceedings of the 8th International Conference on Ubiquitous Computing, 17-21 September, pp. 19-34.

[13] Carles Gomez and Joseph Paradells,(2010) "Wireless Home Automation Networks: A survey of Architectural and Technologies”, IEEE Communication Magzine, June, pp. 92-101

[14] A.J. D. Rathnayaka, V.M. Poddar, (2011)“ Evaluation of Wireless Home Automation Technologies”, Proceedings of the 5th International Conference on Digital Ecosystems and Technologies, May 31, 2011, Daejeon, Korea, pp. 76-81

[15] Frost and Sullivan, "Innovation and Affordability open up home automation to alarge audience" available at http://www.frost.com/prod/servlet/press-release-pag?docid=273090581

[16] The Z-Wave technology available at http://www.Z-wavealliance.org/technology

[17] P. Darbee, “ INSTEON: The Details", 2005-2013 available at www.insteon.com

[18] http://www.radiocomms.com.au/products/42985-Wavenis-Open-Standard-Alliance

[19] https://www.bluetooth.org

[20] http://www.wi-fi.org/

[21] https://www.zigbee.org/

[22] The IEEE 802.15.4 standard available at http://standards.ieee.org/getieee802/download/802.15.4d2009.pdf

[23] IPv6 over Low power WPAN (6lowpan) available at http://datatracker.ietf.org/wg/6lowpan/

[24] http://www.enocean-alliance.org/en/home/

[25] http://www.thetrackit.com/library/UWB\%20Defin.pdf

[26] Dechuan Chen and MeifangWabg,(2006) "A home security ZigBee network for remote monitoring application", Proceedings of IET International Conference on Wireless, Mobile, and Multimedia networks", November 6-9, Hangzhou , China, pp. 1-4

[27] ManaseePatil and S.R.N. Reddy, (2013) "Design and Implementation of Home/Office Automation System based on Wireless Technologies", International Journal of Computer Application, Vol. 69,No.6, October, pp. 19-22

[28] V. Sathya Narayanan1 and S. Gayathri, "Design of Wireless Home automation and security system using PIC Microcontroller", International Journal of Computer Applications in Engineering Sciences, Vol. 3, special issue, pp. 135-140 
International Journal of Computer Networks \& Communications (IJCNC) Vol.6, No.4, July 2014

[29] Woo-Choo Park and Myung-Hyun Yoon, (2006)“ The Implementation of Indoor Location System to Control ZigBee Home Network", Proceedings of International Joint Conference SICR-ICASE”, October 18-21, Busan, South Korea, pp. 2158-2161

[30] Kawamoto, R., Emon, T., Sakata, S., and Youasa, K., (2007)“ Energy efficient sensor control scheme for Home Networks based on DLNA-ZigBee Gateway Architecture”, Proceedings of the First International Global Information Infrastructure Symposium, July 2-6, 2007, Marrakech, Morocco, pp. 73-79

[31] Zhang Shunyang, Xu Du, Jiang Yongping,() Wang Riming, “ Realization of Home Remote Controlnetworks based on ZigBee", Proceedings of the 8th International Conference on Electronic Measurements and Instrumentations, August 16-18, Xiang, China, pp. 4-344-4-348

[32] Jan Hou, Wu Cang dong, Zhongjia Yuan, Jiyuan Tan, " Research of Intelligent Home Security Surveillance System based on ZigBee", Proceedings of the Initial Symposium on Intelligent Information Technology Application Workshops, December 21-22, Shanghai, pp. 554-557

[33] Jianfeng Wu, and Hubin Qin, (2008)“ The deisgn of wireless intelligent home system based on ZigBee", Proceedings of the 11th IEEE International Conference on Communication Technology, November 11-12, HagZhau, pp. 73-76

[34] Chunglong Zhang, Min Zhang, Young Sheng Su, and Weillian Wang, (2012) " Smart home design based on ZigBee wireless sensor network", Proceedings of the 7th International ICST Conference on Communications and networking, Kun Ming, pp. 463-466

[35] Jui-Yu Chang, Tao-Yuan, Min-Hsing Hung, and Yen-Wei Chang, (2007) "A ZigBee based Power Monitoring System with Direct Load Control Capabilities", Proceedings of IEEE International Conference on Networking, Sensing, and Control, April 15-17, London, 2007, pp. 895-900

[36] Ying-Wen Bai and Chi-Huang Hang, " Remote Power ON/OFF control and current measurementfor home electric outlets based on a low-power embedded board and ZigBee Communication”, Proceedings of IEEE International Symposium on Consumer Electronics, April 14-16, Vilamoura, pp.1-4

[37] Jin Sung Byum, BoungjuJeon, Junyoung Noh, and Youngil Kim, “ An Intelligent self-adjusting sensor for smart home services based on ZigBee Communiation", IEEE Transaction on Consumer Eletronics, Vol. 58, No. 3, pp. 799-802

[38] Chihhsiong Shih and Bwo-cheng Liang, “ A model driven software framework for ZigBee based energy saving systems", Proceedings of the 3rd International Conference on Intelligent Systems, modeling and Simulation, February 8-10, Kota Kinabaiu, pp. 487-492

[39] Karia Deepak, JaypalBavisker, Raj Makwana and PanchatNiraj,(2013) “Performance analysis of ZigBee based load control and power monitoring system", Proceedings of the InternationalConference on Advances in Computing, Communications, and Informatics, Myshore, August, 25, 2013, pp. 17791484.

[40] Jieming Zhu, Xuecai, Yucang Yang, and Hang Li, (2010)“ Developing a voice control system forZigBee based home automation", Proceedings of IEEE International Conference on network Infrastructure and Digital Content, September 24-26, Beijing, 2010, pp. 7737-741

[41] Y.B. Krishna and S. Nagendram,(2012)“ ZigBee Based Voice Control System for Smart Home”, International Journal on Computer Technology and Applications, Vol. 3, no. $1 \quad$, pp. 163-168

[42] Gananasekar, A.K., Jayarelu, P., and V. Nagrajan,(2012) "Speech recognition based wireless automation of home with fault identification for physically challenged", Proceedings of International Conference on Communications and Signal Processing, April 4-5 2012, Chennai, pp. 128132

[43] Al Shueili, H. and Sen Gupta G, Mukhopadhyaya, (2001)“ Voice recognition based wireless home automation system", Proceedings of the International Conference on Mechatronics , May 17-19, Kualalumpur, pp. 1-6

[44] Dhawan S. Thakur and Aditi Sharma,(2013)" Voice Recognition Wireless Home Automation System based on ZigBee"IOSR Journal of Electronic and Communication Engineering, Vol. 6, No. 1 May-June 2013, pp. 65-75.

[45] MitaliPatil, AshwiniBedare, and VarshaPacharne,(2013) "The Design and Implementation of VoiceControlled Wireless Intelligent Home Automation System Based on ZigBee", International Journal of Advanced Research in Computer Science and Software Engineering, Vol. 3, No. 4, April 2013, pp. 307-310

[46] T. Kirankumar, and B. Bhavani,(2013)" A Sustainable Automated System for Elderly People Using Voice Recognition and Touch Screen Technology", International Journal of Science andResearch (IJSR), Vol. 2, No. 8, August 2013, pp. 265-267 
International Journal of Computer Networks \& Communications (IJCNC) Vol.6, No.4, July 2014

[47] Y.Usha Devi,(2012) "Wireless Home Automation System Using ZigBee", International Journal of Scientific \& Engineering Research,Volume 3, Issue 8, August, 2012, pp. 1-5

[48] http://www.zigbee.org/About/FAQ.aspx

[49] Rathod K., Parikh, N., and Shah, V.,(2012) "Wireless automation using ZigBee protocols", Proceedings of the 9th International Conference on Wireless and Optical Communication, lndere, September 20-22, 2012, pp.1-5

[50] http://www.skyworksinc.com/downloads/press_room/published_articles/ Microwave_Journal_082009.pdf

[51] http://www.engineerlive.com/content/19359

[52] Simek, M., Fuchs, M, Mraz, L., and Morvek, P.,(2011) "Measurement of LowPAN Network coexistence with Home Microwave Appliances in Laboratory and Home Environments", Proceedings of International Conference on Broadband and Wireless Computing, October 26-28, Bercelona, pp. 292-299

[53] Khusvinder Gill, Shuang-Hua Yang, Fang Yao, and Xin Lu, (2009) "A ZigBee-Based Home Automation System", IEEE Transaction on Consumer Electronics”, Vol. 55, No. 2, May 2009, pp. 422430

[54] Fei Ding, Guangming Song, Jianing Li, and Higuo Song, (2008) “ A ZigBee Based Mesh Network forHome Control", Proceedings of International Workshop on Geoscience and Remote Sensing", Shanghai, Vol. 1, pp. 744-740

[55] Cui Chenguyi, Zhao Guannan, and Jin Mingle,(2010) “A ZigBee based embedded remote control System", Proceedings of the 2nd International Conference on Signal Processing Systems, 5-7 July, Dalian, pp. v3-373-376

[56] NausheenBelim, HarshadaBhambure, Priyanka Kumbhar, and SimranjitTuteja,(2013) "Automate and Secure Your Home Using Zigbee Technology", International Journal of Innovative Research in Computer and Communication Engineering, Vol. 1, No. 1, March 2013, pp. 63-66

[57] Meng-Shiuan Pan, Chia-Hung Tsai, Yu-Chee Tseng,(2009) "The Orphan Problem in ZigBee WirelessNetworks", IEEE Transaction of Mobile Computing,November 2009, Vol. 8 , No. 11, pp. 1573-1584

[58] Xiu-Wei Yang, Jinan, China ; Yuan Xu ; Shu-Bo Qiu,(2011) “A Solution of Orphan Problem in ZigBee Tree Network", Proceedings on the 7th International Conference on Wireless Communication, Networking and Mobile Computing, September 2011, Wuhan, pp. 1-4

[59] Michael Varhola and Milos Drutorvsky,(2007) "ZigBee Based Home Automation Wireless Sensor Networks",ActaElectrotechnica et Informatics, Vol. 7, No. 4, 2007, pp. 1-8

[60] Napolean A., Karthik K., Kamalakannan M., Amarnath M., Nidhin A., (2013) "Implementation of ZigBee based Home Automation System Using Voice Control", International Journal of Engineering Research and Technology, Vol. 2, No. 5, May, pp. 723-733 